\title{
Riqueza y distribución de equinodermos en los arrecifes rocosos de Punta Amapala y Los Cóbanos, EI Salvador
}

Johanna Segovia $^{1,2}$, Gilma Guerra ${ }^{1}$ \& Fernanda Ramos ${ }^{1}$

1. Universidad de El Salvador, Final Ave. Estudiantes Héroes y Mártires del 30 de julio, Ciudad Universitaria. San Salvador, El Salvador.

2. Instituto de Ciencias del Mar y Limnología, UES. Final Ave. Estudiantes Héroes y Mártires del 30 de julio, Ciudad Universitaria. San Salvador, El Salvador; jsegovia.icmares@ues.edu.sv

Recibido 09-I-2017. Corregido 05-II-2017. Aceptado 23-VI-2017.

\begin{abstract}
Richness and distribution of Echinoderms in rocky reefs at Punta Amapala and Los Cobanos, El Salvador. In El Salvador, taxonomic and distribution studies of echinoderms are scarce, and most of them are from Los Cóbanos. This research aims to explore the richness and abundance of echinoderms associated with shallow rocky reefs of El Salvador, as well as their relationship between distribution and abiotic variables. Sampling was carried out in Los Cóbanos and Punta Amapala, on the Pacific coast of El Salvador; between February and April 2016, with three-hour workdays during low tide. The sampling techniques used were wandering dive and three transects of $30 \mathrm{~m}$ in length. The relationship between species and abiotic variables (temperature, salinity, coast distance and roughness) was identified with canonical correspondence analysis. We report seven species in five orders. The temperature and coast distance are strongly related to the distribution of organisms. Holothuria (Halodeima) kerfersteini and Echinometra vanbrunti are related to high temperature and greater distance from the coast; however, Ophiocoma aethiops, O. alexandri and Ophiothrix rudis are conditioned by low temperature and shorter coast distance. Rev. Biol. Trop. 65(Suppl. 1): S92-S100. Epub 2017 November 01.
\end{abstract}

Key words: El Salvador; rocky reef; echinoderms; spatial distribution.

Los equinodermos de aguas someras son organismos diversos en regiones tropicales y subtropicales; sin embargo, su diversidad y abundancia disminuye en latitudes altas (Pawson, 2007). A nivel mundial, se registran aproximadamente 7550 especies vivientes (Zhang, 2013), divididas en cinco clases: crinoideos (Crinoidea, 700 especies), estrellas de mar (Asteroidea, 1800 especies), ofiuroideos (Ophiuroidea, 2000 especies), erizos de mar (Echinoidea, 900 especies) y pepinos de mar (Holothuroidea, 1200 especies) (SolísMarín \& Laguarda-Figueras, 2010). Los trabajos de equinodermos en el Pacífico Oriental Tropical, se han enfocado, de manera general, en aspectos taxonómicos, diversidad y patrones de distribución (Caso, 1978, 1980;
Caso, Laguarda-Figueras, Solís-Marín, OrtegaSalas \& Durán-González, 1994; Reyes-Bonilla, 1995; Holguín-Quiñónez, Wright López, \& Solís-Marín, 2000).

En El Salvador, los estudios han sido intermitentes, realizando mayor esfuerzo en la identificación de especies. Hasta la fecha, se han registrado 60 especies, distribuidas en cuatro clases. La clase de mayor representatividad es Holothuroidea (19 spp.), seguido por Ophiuroidea (18 spp.), Echinoidea (15 spp.) y Asteroidea (8 spp.) (Barraza \& Hasbún, 2005; Carballo \& Pocasangre, 2007; Alvarado et al., 2010; Alvarado \& Solís-Marín, 2013). De estas especies, 13 se reportan para sustratos rocosos en aguas someras menores a $3 \mathrm{~m}$ de profundidad en El Pital, Los Cóbanos, Maculís 
y Solymar (Barraza \& Hasbún, 2005). En 2009, se evidenció alta extracción y comercialización de Holoturoideos, es por ello, que fueron incluidos dentro del Listado Oficial de Especies de Vida Silvestre Amenazada o en Peligro de Extinción de El Salvador, bajo la categoría de amenazados (Ministerio de Medio Ambiente y Recursos Naturales, 2015).

La distribución de los equinodermos en ambientes costeros es influenciada por aspectos físicos y químicos. Las mareas condicionan principalmente el hábitat, los oleajes fuertes golpean los litorales o acantilados rocosos formando cavernas y terrazas de abrasión, donde se encuentran principalmente erizos de la familia Diadematidae (Diadema mexicanum) y en ocasiones, algunos ofiuroideos como Ophioderma teres, $O$. panamense y $O$. alexandri, $O$. aethiops (Neira \& Cantera, 2005). Caso et al. (1994) describe que en términos generales, se conoce que la clase Ophiuroidea está condicionada al tipo de sustrato y a la salinidad; mientras que la clase Asteroidea, muestra mayor sensibilidad a las variaciones de salinidad y la temperatura; la ocurrencia de la clase Echinoidea se condiciona a la salinidad y el tipo de sustrato, pero la clase Holothuroidea tiene mayor afinidad con la temperatura.

Existen grupos importantes para el monitoreo de arrecifes, por ejemplo, los erizos; que tienen una activa participación en los procesos de bioerosión del ecosistema, benefician el reclutamiento de corales y asentamiento de epifauna (Bak, 1990; Edmunds \& Carpenter, 2001; Herrera-Escalante, López-Pérez \& Leyte-Morales, 2005; Alvarado \& Solis-Marín, 2013). Además, contribuyen a la desintegración del sustrato, lo que incrementa la complejidad del hábitat (Lozano-Cortés, Londoño-Cruz, \& Zapata, 2011).

El objetivo de este trabajo es conocer la riqueza y abundancia de los equinodermos asociados a los arrecifes rocosos de poca profundidad de El Salvador; así como, la relación que existe entre su distribución y las variables abióticas del ecosistema.

\section{MATERIALES Y MÉTODOS}

Sitio de estudio: El Salvador se ubica en el centro de Mesoamérica, y posee $321 \mathrm{~km}$ de costa en la vertiente del Océano Pacífico, al noroeste del país, predominan extensas playas arenosas y lagunas costeras; mientas que al suroeste, la costa presenta golfos, penínsulas, acantilados y arrecifes rocosos (GierloffEmden, 1976). Los sitios de muestreo fueron Los Cóbanos, la cual es un Área Natural Protegida (ANP) (1331'25.9" N y 8948'21.9" $\mathrm{O}$ ); que se caracteriza por la presencia de arrecifes rocosos con formaciones coralinas (Reyes-Bonilla \& Barraza, 2003). El segundo sitio fue Punta Amapala $\left(13^{\circ} 09^{\prime} 21.6^{\prime \prime} \mathrm{N}\right.$ y $87^{\circ} 54^{\prime} 9.2$ " O); que cuenta con comunidades algales, de octocorales y esponjas (Domínguez, 2011; Segovia, 2012) (Fig. 1).

Fase de campo y laboratorio: Los muestreos se realizaron entre los meses de febrero a abril (2016), con jornadas de trabajo de tres horas durante marea baja. La colecta de especies se efectuó con buceo libre, utilizando la técnica del buceo errante, recorriendo en cada área un espacio de $1 \mathrm{~km}^{2}$. Las abundancias y variables físico - químicas se tomaron en tres transecto de $30 \mathrm{~m}$ de longitud paralelos a la costa, ubicados a una distancia de 100, 200 y $300 \mathrm{~m}$. Los transectos se recorrieron con una $\mathrm{T}$ de PVC $(1 \mathrm{~m})$, donde se revisó bajo las rocas del área y teniendo el cuidado de colocarlas en su posición original, se contabilizó por especie el número de individuos. Las especies fueron colectadas con bolsas herméticas con agua de mar, trasladadas al laboratorio en hieleras y adormecidos a bajas temperaturas antes de ser fijados y preservados. Los ejemplares se fijaron en formaldehído diluido en agua de mar $(4 \%)$, y preservados en alcohol etílico al $70 \%$. La identificación de las especies colectadas se realizó utilizando las claves taxonómicas de Fell (1960) y Solís-Marín, ArriagaOchoa, Laguarda-Figueras, Frontana-Uribe, \& Durán-González (2009), así como también las guías ilustradas de Kertitch \& Bertsh (2007) y los artículos de Alvarado, Reyes-Bonilla \& 


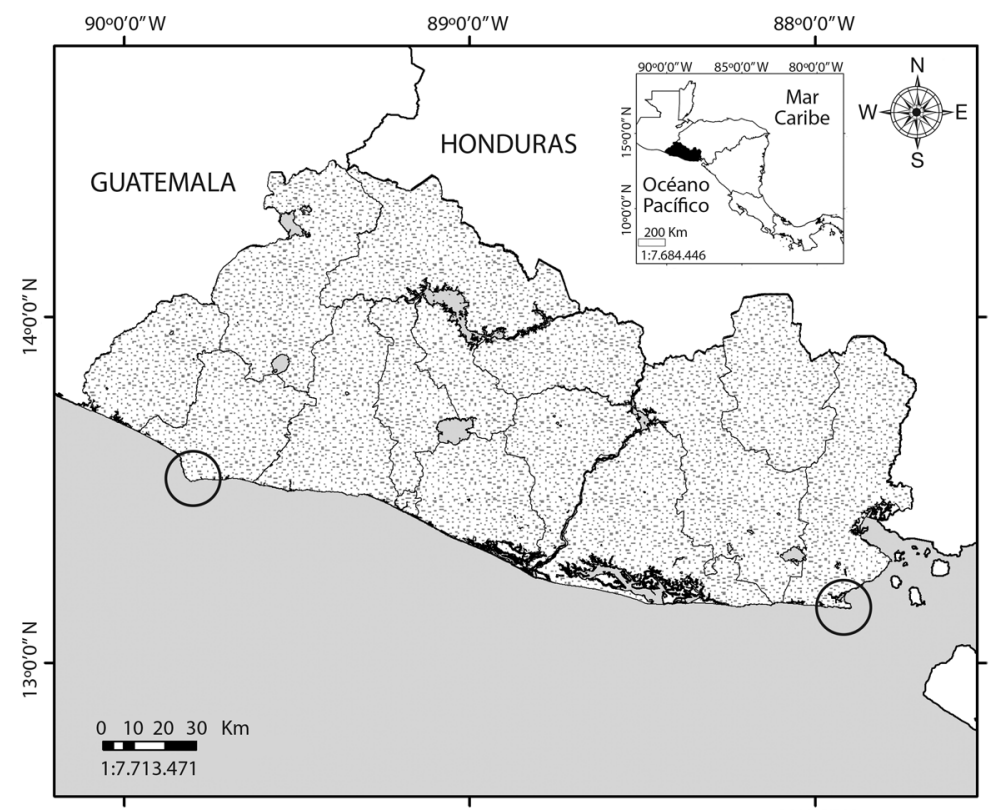

Fig. 1. Localización de los sitios de muestreo: Los Cóbanos y Punta Amapala, El Salvador, Centroamérica.

Fig. 1. Localization of sampling stations: Los Cóbanos and Punta Amapala, El Salvador, Centroamérica.

Benítez-Villalobos, (2015) y Granja-Fernández et al. (2014). La nomenclatura fue actualizada de acuerdo a la World Register of Marine Species (www.marinespecies.org). Los especímenes fueron depositados en la Colección Científica de Invertebrados Acuáticos del Instituto de Ciencias del Mar y Limnología de El Salvador (ICMARESUES-CI).

Las variables abióticas se registraron con un sensor PCSTestr35 para temperatura $\left(0.1 \pm 0.5^{\circ} \mathrm{C}\right)$, y salinidad (0.01). La distancia de costa con una cinta métrica de $30 \mathrm{~m}$ y la rugosidad (IR) con una cadena de $10 \mathrm{~m}$ de largo; la cual, se puso sobre el contorno del sustrato y se midió la distancia lineal de la misma con cinta métrica. Este resultado se inserto en la siguiente fórmula: $I R=1$-(distancia lineal de cadena/distancia total de cadena).

Análisis estadístico: La relación entre especies y variables abióticas del ecosistema se realizó con Análisis de Correspondencia Canónica (ACC). Para el ACC se comprobó la normalidad y homoscedasticidad de los datos. El software utilizado fue PAST 3.12.

\section{RESULTADOS}

Riqueza y similitud de especies: Los equinodermos de arrecifes rocosos de aguas someras (hasta $300 \mathrm{~m}$ de distancia de costa) registrados en Los Cóbanos y Punta Amapala fueron siete especies en seis géneros, seis familias y cinco ordenes. La clase Ophiuroidea presentó tres especies, seguida de las clases Echinoidea y Holothuroidea, ambas con dos especies (Cuadro 1). Las localidades comparten la presencia de seis especies, y se diferencia cada sitio por una especie. En el caso de Los Cóbanos fue Diadema mexicanum y en Punta Amapala fue Afrocucumis ovulum. Estos registros representan el 54\% de los reportes hechos en el país para litoral rocoso de aguas someras a menos de $3 \mathrm{~m}$.

Abundancia y distribución espacial: En los transectos se registraron cinco especies en total: Ophiocoma aethiops, O. alexandri, Ophiothrix rudis, Echinometra vanbrunti y Holothuria (Halodima) kefersteinii. La especie con mayor abundancia fue E. vanbrunti 
CUADRO 1

Listado sistemático de equinodermos de Los Cóbanos y Punta Amapala

TABLE 1

List systematic of echinoderm from Los Cóbanos and Punta Amapala

\author{
Clase Ophiuroidea \\ Orden Ophiurida \\ Familia Ophiocomidae \\ Ophiocoma alexandri Lyman, 1860 \\ Ophiocoma aethiops Lütken, 1859 \\ Familia Ophiotrichidae \\ Ophiothrix rudis Lyman, 1874 \\ Clase Echinoidea \\ Orden Camarodonta \\ Familia Echinometridae \\ Echinometra vanbrunti A. Agassiz, 1863 \\ Orden Diadematoida \\ Familia Diadematidae \\ Diadema mexicanum A. Agassiz, 1863
}

Clase Holothuroidea

Orden Aspidochirotida

Familia Holothuriidae

Holothuria (Halodeima) kefersteinii Selenka, 1867

Orden Dendrochirotida

Familia Sclerodactylidae

Afrocucumis ovulum Selenka, 1867
(106 individuos), presente en ambos sitios de estudio, seguida por $O$. aethiops y $O$. alexandri con 27 y 25 individuos respectivamente, de igual forma registrada en ambos sitios. Las menos abundantes fueron $H$. (Halodei$m a)$ kefersteinii (6 individuos) y $O$. rudis (3 individuos), esta última especie se registró únicamente en Los Cóbanos (Cuadro 2). $\mathrm{La}$ abundancia de equinodermos para ambos sitios fue 167 individuos en $180 \mathrm{~m}^{2}$, con densidades de 1 indv $\mathrm{m}^{-2}$. El arrecife rocoso de Punta Amapala presentó mayor densidad (1.22 indv $\mathrm{m}^{-2}$ ) que el arrecife de coral Los Cóbanos (0.63 indv $\mathrm{m}^{-2}$ ) (Cuadro 3).

Punta Amapala registró mayor temperatura $\left({ }^{\circ} \mathrm{C}\right)$ y densidad de $E$. vanbrunti y $O$. aethiops; sin embargo, Los Cóbanos posee mayor densidad de $O$. alexandri. El ACC (trance 0.392, $p=0.04)$ agrupa el $70 \%$ de la variación en el primer eje $(p=0.007)$; el cual, refleja que E. vanbrunti y $H$. keferstenii se relaciona con sitios de mayor temperatura $\left(31^{\circ} \mathrm{C}\right)$ e intermedia salinidad $(31 \%$, aunque E. vanbrunti con puntos de mayor rugosidad; como en Punta Amapala. O. aethiops, O. rudis y $O$. alexandri se encuentran en sitios de menor temperatura $\left(27\right.$ a $\left.28^{\circ} \mathrm{C}\right)$ de Los Cóbanos; en el caso de

CUADRO 2

Individuos identificados por transecto en cada uno de los sitios de estudio. Co1: Los Cóbanos, Transecto 1; Co2: Los Cóbanos, Transecto 2; Co3: Los Cóbanos, Transecto 3. PA1: Punta Amapala, Transecto 1; PA2: Punta Amapala, Transecto 2; PA3: Punta Amapala, Transecto 3

TABLE 2

Individuals identified by transect at each of the study sites. Co1: Los Cóbanos, Transect 1; Co2: Los Cóbanos, Transect 2; Co3: Los Cóbanos, Transect 3. PA1: Punta Amapala, Transect 1; PA2: Punta Amapala, Transect 2;

PA3: Punta Amapala, Transect 3

\begin{tabular}{|c|c|c|c|c|c|c|}
\hline Sitio & Transecto & $\begin{array}{c}\text { Echinometra } \\
\text { vanbrunti }\end{array}$ & $\begin{array}{c}\text { Holothuria (Halodeima) } \\
\text { kefersteinii }\end{array}$ & $\begin{array}{l}\text { Ophiocoma } \\
\text { aethiops }\end{array}$ & $\begin{array}{l}\text { Ophiocoma } \\
\text { alexandri }\end{array}$ & $\begin{array}{l}\text { Ophiothrix } \\
\text { rudis }\end{array}$ \\
\hline \multirow[t]{3}{*}{ Los Cóbanos } & Col & 6 & 0 & 0 & 5 & 1 \\
\hline & $\mathrm{Co} 2$ & 17 & 0 & 4 & 6 & 0 \\
\hline & $\mathrm{Co} 3$ & 1 & 1 & 7 & 7 & 2 \\
\hline \multirow[t]{4}{*}{ Punta Amapala } & PA1 & 18 & 0 & 6 & 7 & 0 \\
\hline & PA2 & 48 & 2 & 10 & 0 & 0 \\
\hline & PA3 & 16 & 3 & 0 & 0 & 0 \\
\hline & & 106 & 6 & 27 & 25 & 3 \\
\hline
\end{tabular}


CUADRO 3

Densidades por especie (indv $\mathrm{m}^{-2}$ ) en Los Cóbanos y Punta Amapala, El Salvador

TABLE 3

Densities per species (indv $\mathrm{m}^{-2}$ ) of Los Cóbanos and Punta Amapala, El Salvador

\begin{tabular}{lccccc}
\multicolumn{1}{c}{ Sitio de estudio } & $\begin{array}{c}\text { Echinometra } \\
\text { vanbrunti }\end{array}$ & $\begin{array}{c}\text { Holothuria (Halodeima) } \\
\text { kefersteinii }\end{array}$ & $\begin{array}{c}\text { Ophiocoma } \\
\text { aethiops }\end{array}$ & $\begin{array}{c}\text { Ophiocoma } \\
\text { alexandri }\end{array}$ & $\begin{array}{c}\text { Ophiothrix } \\
\text { rudis }\end{array}$ \\
Los Cóbanos & $0.27 \pm 0.091$ & $0.01 \pm 0.006$ & $0.12 \pm 0.039$ & $0.2 \pm 0.011$ & $0.03 \pm 0.011$ \\
Punta Amapala & $0.91 \pm 0.199$ & $0.06 \pm 0.017$ & $0.18 \pm 0.056$ & $0.08 \pm 0.045$ & 0 \\
\hline
\end{tabular}

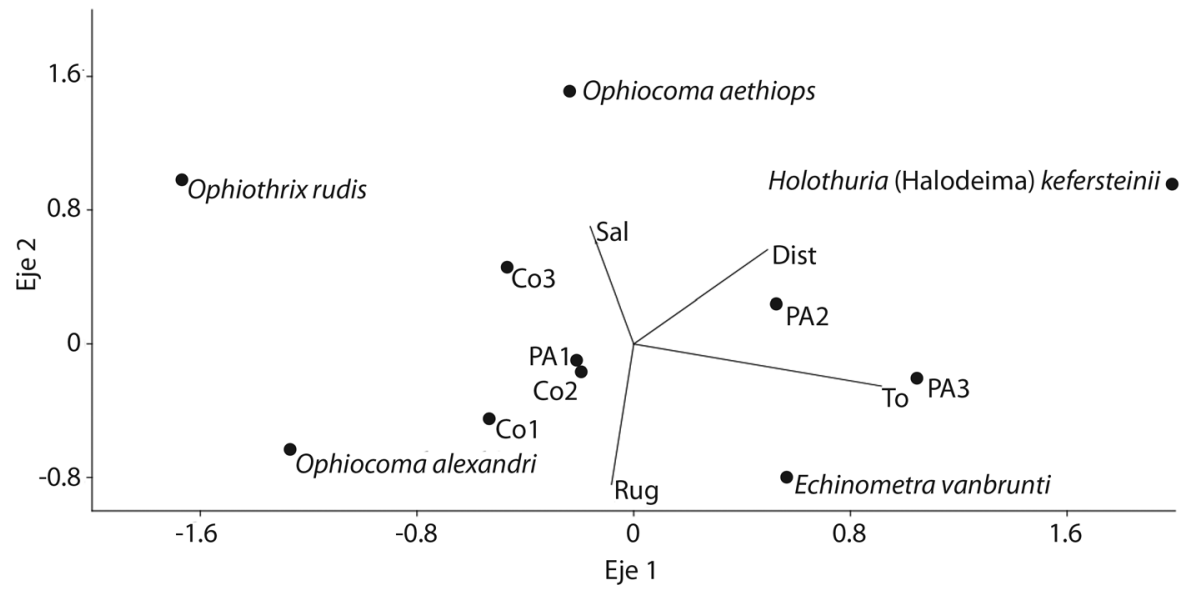

Fig. 2. Análisis de Correlación Canónica que muestra la relación entre variables bióticas y abióticas. Dist: Distancia de costa; To: Temperatura; Sal: Salinidad; Rug: Rugosidad; Co1: Transecto 1, Los Cóbanos; Co2: Transecto 2, Los Cóbanos; Co3: Transecto 3, Los Cóbanos; PA1: Transecto 1, Punta Amapala; PA2: Transecto 2, Punta Amapala; PA3: Transecto 3, Punta Amapala.

Fig. 2. Canonical Correlation Analysis showing the relationship between biotic and abiotic variables. Dist: Coastal distance; $\mathrm{T}^{\mathrm{o}}$ : Temperature; Salt: Salinity; Rug: Roughness; Co1: Transect 1, Los Cóbanos; Co2: Transect 2, Los Cóbanos; Co3: Transect 3, Los Cóbanos; PA1: Transect 1, Punta Amapala; PA2: Transect 2, Punta Amapala; PA3: Transect 3, Punta Amapala.

$O$. aethiops, se relaciona con puntos de mayor salinidad $(33 \%$ y $O$. alexandri con áreas de mayor rugosidad (Fig. 2). Sin embargo, las variables de salinidad y rugosidad, son vectores cercanos al segundo eje, que resume el $2 \%$, pero no posee significancia estadística $(\mathrm{p}=0.452)$.

\section{DISCUSIÓN}

Para El Salvador se han reportado 13 especies de equinodermos en litorales rocosos, de los cuales en esta investigación se reportan siete. La clase Ophiuroidea presentó una especie más que la clases Echinoidea y
Holothuroidea. Contrario a Alvarado \& Chiriboga (2008), que observaron más especies en Echinoidea para la Isla del Coco, así mismo, Zamorano \& Leyte-Morales (2009) en Zihuatanejo y Acapulco (México). Sin embargo, Alvarado \& Fernández (2005) reportan a Holothuroidea con mayor diversidad en Marino Ballena (Costa Rica). Finalmente, Alvarado, Guzmán y Breedy (2012) registran similar número de especies en las clases Asteroidea, Echinoidea y Holothuroidea para las islas del Golfo de Chiriqui (Panamá).

Los arrecifes de El Salvador en su mayoría son rocosos, excepto en Los Cóbanos, donde 
encontramos comunidades monoespecificas de Porites lobata; ambos hábitat reportan baja riqueza asociada de equinodermos, lo que coincide con otros sitios de la región; ya que se reconoce que son ecosistemas poco diversos en composición de equinodermos en aguas someras, en comparación con otros arrecifes del mundo (Alvarado \& Chiriboga, 2008). Generalmente, los sitios que presentan mayores diversidades en este grupo, son áreas con tasas bajas de sedimentación, respondiendo a los requerimientos de selección de hábitat de las especies (Alvarado \& Fernández, 2005).

Las especies observadas en Los Cóbanos y Punta Amapala son comunes en el Pacífico Oriental Tropical. Por ejemplo, al igual que Brusca (1980) y Alvarado \& Fernández (2005), se registró mayor abundancia de organismos en Ophiocoma aethiops y O. alexandri, las cuales, son especies que se describen como abundantes en las costas rocosas de la zona media intermareal. Mientras, Holothuria (Halodeima) keffersteinii posee mayor afinidad al Indo-Pacífico (Hickman, 1998; Miskelly, 2002; Alvarado \& Fernández, 2005). Otra especie abundante en el estudio fue Echinometra vanbrunti, lo que coincide con Alvarado et al. (2012) para las islas del Golfo de Chiriqui (Panamá).

La frecuencia de $O$. aethiops y $O$. alexandri está condicionada por las variables abióticas de rugosidad de sustrato y salinidad, presentando en el caso de $O$. aethiops, mayor número de individuos en sustratos con alta rugosidad. Lo que coincide con los resultados obtenidos por Caso et al. (1994) en la clase Ophiuroidea, donde se evidencia que la presencia de la clase, estuvo más condicionada al tipo de sustrato que a las variaciones de la temperatura en Bahía de Mazatlán (Sinaloa, México).

Las altas abundancias de estos organismos son similares en proporción a las reportadas por Carballo \& Pocasangre (2007) para Los Cóbanos, donde los autores atribuyen su abundancia a la poca capacidad móvil, y su habilidad de utilizar grietas, agujeros, espacios entre las rocas y otros huecos naturales como refugio; lo cual facilita su observación y contabilización durante los muestreos en campo. También existe coincidencia con los resultados de Alvarado \& Fernández (2005), quienes detectaron mayores abundancias de estas dos especies en el Parque Nacional Marino Ballena, Costa Rica. La ocurrencia frecuente de estas especies en sustratos rocosos y coralinos coincide con lo reportado por Barraza (2005), Segovia \& Navarrete (2007), Carballo \& Pocasangre (2007), Alvarado et al. (2010), Alvarado \& Solís-Marín (2013) para la costa salvadoreña.

La densidad de $O$. aethiops en Punta Amapala (0.18 indv $\left.\mathrm{m}^{-2}\right)$ y Los Cóbanos (0.12 indv $\left.\mathrm{m}^{-2}\right)$, fue el doble a la reportada en Los Cóbanos por Carballo \& Pocasangre (2007), podemos argumentar que esto se debe a la temporada de crecimiento-maduración de la especie; la cual, según Aguilar-Duarte (2011) se encuentra en diciembre y de febrero a abril. $O$. alexandri, describe un patrón muy similar a $O$. aethiops para Los Cóbanos, mostrando mayor densidad $\left(0.2 \mathrm{indv} \mathrm{m}^{-2}\right)$ que la reportada por Carballo \& Pocasangre (2007); sin embargo, se observó baja densidad para Punta Amapala (0.08 indv $\left.\mathrm{m}^{-2}\right)$. Ophiothrix rudis se observó únicamente para Los Cóbanos, con densidad baja, en relación a las otras especies $\left(0.03 \mathrm{indv} \mathrm{m}^{-2}\right)$, y es probable que la característica del hábitat de presentar piedras sueltas en el área, proporcionan un hábitat idóneo para esta especie, por su tipo de alimentación. Esta dinámica en el hábitat de la especie es similar a la descrita por Carballo \& Pocasangre (2007).

E. vanbrunti fue la especie que presentó mayor densidad de individuos para ambos sitios, siendo más alta en Punta Amapala (0.91 indv $\left.\mathrm{m}^{-2}\right)$, su abundancia y distribución se relaciona a altas temperaturas $\left(29\right.$ a $\left.31^{\circ} \mathrm{C}\right)$ y a distancias mayores de $200 \mathrm{~m}$ desde la costa. Lo que coincide con lo reportado por Caso et al. (1994), quien refiere a los fondos rocosos y arenosos expuestos al oleaje como hábitats idóneos para la especie. Así mismo, describe los máximos valores de abundancia para la Bahía de Mazatlán entre 29.5 a $31.4^{\circ} \mathrm{C}$. H. (Halodeima) kerfersteinii se registró sólo para Punta Amapala y con densidad baja, sin embargo su abundancia se relacionó a valores altos de temperatura $\left(30^{\circ} \mathrm{C}\right)$ y a distancias 
mayores de $200 \mathrm{~m}$ desde la costa. Sin embargo, Carballo \& Pocasangre (2007) la reportan como una de las especies con mayor densidad para Los Cóbanos.

La información generada sobre la relación entre factores físico-químicos y la biodiversidad de equinodermos para el país, ayuda a los gestores ambientales a tener conocimiento para sustentar herramientas y estrategias de manejo para este ecosistema. Sin embargo, es recomendable seguir con los esfuerzos que incluyan análisis estacionales, relaciones ecológicas, alimentación y ciclos de vida de las especies. Así como, variables de sedimentación, amplitud de marea, acción de las olas y viento; los cuales determinan la supervivencia de los organismos del grupo.

\section{AGRADECIMIENTOS}

La presente investigación se realizó con el apoyo en campo de estudiantes de Licenciatura en Biología de la Universidad de El Salvador (UES), en modalidad de voluntariado y servicio social en el Instituto de Ciencias del Mar y Limnología (ICMARES). Además, los autores agradecen a Juan José Alvarado por su invitación a participar en este suplemento especial. Los resultados son datos obtenidos del Programa de Monitoreo de Arrecifes Rocosos y de Coral del ICMARES, UES; con el apoyo logístico y financiero de la Facultad de Ciencias Naturales y Matemática y la Secretaría de Investigación Científica (CIC UES 13.10 "Salud ecológica de los arrecifes de El Salvador ante el Cambio Climático").

\section{RESUMEN}

El trabajo busca conocer la riqueza y abundancia de los equinodermos asociados a los arrecifes rocosos de poca profundidad de El Salvador; así como, la relación que existe entre su distribución y las variables abióticas del ecosistema. Los muestreos se realizaron en Los Cóbanos y Punta Amapala de El Salvador, entre febrero a abril de 2016, con jornadas de trabajo de tres horas durante marea baja. Las técnicas de muestreo utilizadas fueron buceo errante y tres transectos de $30 \mathrm{~m}$ de longitud. La relación entre especies y variables abióticas (temperatura, salinidad, distancia de costa y rugosidad) se realizó con análisis de correspondencia canónica. Se obtuvieron siete especies en cinco ordenes. Las variables de temperatura y distancia desde costa se relacionan fuertemente a la distribución de los organismos. Holothuria (Halodeima) kerfersteini y Echinometra vanbrunti se relaciona con alta temperatura y mayor distancia desde la costa; sin embargo, Ophiocoma aethiops, $O$. alexandri y Ophiothrix rudis están condicionadas por baja temperatura y menor distancia desde la costa.

Palabras clave: El Salvador; arrecifes rocosos; equinodermos; distribución espacial.

\section{REFERENCIAS}

Aguilar-Duarte, C. (2011). Comparación del patrón reproductivo entre Ophiocoma alexandri y Ophiocoma aethiops (Echinodermata: Ophiuroidea) y su relación con las variables ambientales temperatura, luminosidad, horas de luz, pluviosidad, clorofilas y salinidad (Tesis de Maestría). Universidad del Mar. Oaxaca, México.

Alvarado, J.J. \& Fernández, C. (2005). Equinodermos del Parque Nacional Marino Ballena, Pacífico, Costa Rica. Revista de Biología Tropical, 53, 275-284.

Alvarado, J.A. \& Chiriboga, A. (2008). Distribución y abundancia de equinodermos en las aguas someras de la Isla del Coco, Costa Rica (Pacífico Oriental). Revista de Biología Tropical, 56, 99-111.

Alvarado, J.J. \& Solís Marín, F.A. (2013). Echinoderm research and diversity in Latin America. In: J.J Alvarado and F.A. Solís-Marín (Eds.), Echinoderm Research and Diversity in Latin America (pp. 1-9). Berlin: Springer.

Alvarado J.J., Solís-Marín, F. \& Ahearn, G.C. (2010). Echinoderm (Echinodermata) diversity in the Pacific coast of Central America. Marine Biodiversity, $40,45-56$.

Alvarado J.J., Guzman, H.M. \& Breedy, O. (2012). Distribution and diversity of echinoderms (Asteroidea, Echinoidea, Holothuroidea) in the islands of the Gulf of Chiriqui, Panama. Revista de Biología Marina y Oceanografia, 47, 13-22.

Alvarado, J.J., Reyes-Bonilla, H. \& Benítez-Villalobos, F. (2015). Diadema mexicanum, erizo de mar clave en los arrecifes coralinos del Pacífico Tropical Oriental: lo que sabemos y perspectivas futuras (Diadematoida: Diadematidea). Revista de Biología Tropical, 63 (Supl. 2), 135-157.

Bak, R. (1990). Patterns of echinoid bioerosion in two Pacific coral reef lagoons. Marine Ecology Progress Series, 66, 267-272. 
Barraza, J. E \& Hasbún, C.R. (2005). Los equinodermos (Echinodermata) de El Salvador. Revista de Biología Tropical, 53, 139-146.

Brusca, R.C. (Eds.) (1980). A handbook to the common intertidal invertebrates of the Gulf of California, 2d(revised). Tucson, University of Arizona Press.

Carballo, R.E. \& Pocasangre, X.M. (2007). Composición y Estructura de la Fauna Intermareal de Equinodermos en el Sistema Arrecifal Rocoso Los Cóbanos, Departamento de Sonsonate, El Salvador (Tesis de Licenciatura). Universidad de El Salvador, San Salvador, El Salvador.

Caso, M.E. (1978). Ciencia y técnica de los equinodermos en relación con el hombre. Primera parte. Anales del Centro de Ciencias del Mar y Limnología, 5, 255-286.

Caso, M.E. (1980). Los equinodermos del Pacífico de México. Parte Tercera. Orden Clypeasteroidea. Anales del Centro de Ciencias del Mar y Limnología, 4, $1-252$.

Caso, M.E., Laguarda-Figueras, A., Solís-Marín, F.A., Ortega-Salas, A. \& Durán-González, A.L. (1994). Contribución al conocimiento de la ecología de las comunidades de equinodermos de la Laguna de Términos, Campeche, México. Anales del Centro de Ciencias del Mar y Limnología, 21, 67-85.

Domínguez, J.P. (2011). Caracterización biofisica del área marina frente a playa Las Tunas, playas Negras, playas Blancas, playa Maculís, y Las Mueludas, Municipio de Conchagua, Departamento de La Unión, El Salvador (Reporte técnico). San Salvador, El Salvador. USAID/IMCCW.

Edmunds, P.J. \& Carpenter, R.C. (2001). Recovery of Diadema antillarum reduces macroalgal cover and increases abundances of juvenile corals on a Caribbean reef. Proceedings of National Academy of Sciences, 98, 5067-5071.

Fell, H.B. (1960). Synoptic Keys to the Genera of Ophiuroidea, Zoology Publications, Victoria University of Wellington, New Zealand, 26, 1-44.

Gierloff-Emden, H.G. (Eds.) (1976). La Costa de El Salvador: monografia-oceanográfica. Dirección de Publicaciones, Ministerio de Educación, San Salvador, El Salvador.

Granja-Fernández, R., Herrero-Pérezrul, M.D., LópezPérez, R.A, Hernández, L., Rodríguez-Zaragoza, F., Wallace, R. \& Pineda-López, R. (2014). Ophiuroidea (Echinodermata) from coral reefs in the Mexican Pacific. ZooKeys, 406, 101-145.

Herrera-Escalante, T., López-Pérez, R.A. \& Leyte-Morales. G.E. (2005). Bioerosion caused by the sea urchin Diadema mexicanum (Echinodermata: Echinoidea) at Bahías de Huatulco Western Mexico. Revista de Biología Tropical, 53, 263-273.

Hickman, C.P. (Eds.) (1998). A Field Guide to Sea Stars and other Echinoderms of Galápagos. Sugar Spring, Virginia.

Holguín-Quiñonez, O., Wright López, H. \& Solís-Marín, F.A. (2000). Asteroidea, Echinoidea y Holothuroidea en fondos someros de la Bahía de Loreto, Baja California Sur, México. Revista de Biología Tropical, $48,749-757$.

Kertitch, A. \& Bertsh, H. (2007). Sea of Cortez Marine Invertebrates: A guide for the Pacific coast, México to Peru ( $2^{\text {nd }}$ edition). Monterrey, California.

Lozano-Cortés, D.F., Londoño-Cruz, E. \& Zapata, F.A. (2011). Bioerosión de Sustrato Rocoso por erizos en Bahía Málaga (Colombia), Pacífico Tropical. Revista de Ciencias, 15, 9-22.

Ministerio de Medio Ambiente y Recursos Naturales. (2015). Listado Oficial de Especies de Vida Silvestre Amenazada o en Peligro de Extinción de El Salvador. Diario Oficial, Número 181, Tomo No 409, 45-65.

Miskelly, A. (Eds.) (2002). Sea Urchins of Australia and the Indo-Pacific. Capricornia, Sydney, Australia.

Neira, R \& Cantera, J. (2005). Composición taxonómica y distribución de las asociaciones de equinodermos en los ecosistemas litorales del Pacifico Colombiano. Revista de Biología Tropical, 53, 195-206.

Pawson, D. L. (2007). Phylum Echinodermata. Zootaxa, $1668,749-764$

Reyes-Bonilla, H. (1995). Asteroidea and Echinoidea (Echinodermata) from Isla San Benedicto, Revillagigedo Archipelago, Mexico. Revista de Investigación Científica, 6, 29-38.

Reyes-Bonilla, H. \& Barraza, J.E. (2003). Corals and associated marine communities from El Salvador. In: J. Cortés (Eds.), Coral Reefs of Latin America (pp. 351-360). The Netherlands: Springer.

Segovia, J. (2012). Comunidades de Octocorales (Cnidaria: Octocorallia) en El Salvador, América Central (Tesis de Magister Scientiae). Escuela de Biología, Universidad de Costa Rica, San José, Costa Rica.

Segovia J. \& Navarrete, M.T. (2007). Biodiversidad a nivel de ecosistema en parches de corales hermatipicos (Porites lobata, Pocillopora sp.) en la playa Los Cóbanos, Sonsonate (Tesis de Licenciatura). Escuela de Biología, Universidad de El Salvador, San Salvador, El Salvador.

Solís-Marín, F.A., Arriaga-Ochoa, J.A., Laguarda-Figueras, A., Frontana-Uribe, S.C. \& Durán-González, A. (2009). Holoturoideos (Echinodermata: Holothuroidea) del Golfo de California (Informe técnico). 
Comisión Nacional para el Conocimiento y Uso de la Biodiversidad e Instituto de Ciencias del Mar y Limnología, Universidad Autónoma de México. D. F., México.

Solís-Marín, F.A. \& Laguarda-Figueras, A. (2010). Los equinodermos del estado de Yucatan. In: Durán R. \& M. Méndez (Eds), Biodiversidad y Desarrollo Humano en Yucatán (pp. 248-249). CICY, PPD-FMAM, CONABIO, SEDUMA, DF, México.
Zamorano, P. \& Leyte-Morales, G.E. (2009). Equinodermos asociados a formaciones arrecifales en Zihuatanejo y Acapulco, Guerrero, México. Boletín de Investigaciones Marinas y Costeras, 38, 7-28.

Zhang, Z.Q. (2013). Animal biodiversity: An update of classification and diversity in 2013. In: Z.Q. Zhang (Eds.), Animal Biodiversity: An Outline of Higherlevel Classification and Survey of Taxonomic Richness (Addenda 2013). Zootaxa, 3703, 5-11. 